Copyright 2008 Society of Photo-Optical Instrumentation Engineers.

This paper was published in SPIE Medical Imaging (2008), 6914, and is made available as an electronic reprint with permission of SPIE. One print or electronic copy may be made for personal use only. Systematic or multiple reproduction, distribution to multiple locations via electronic or other means, duplication of any material in this paper for a fee or for commercial purposes, or modification of the content of the paper are prohibited. 


\title{
Bi-planar 2D-to-3D Registration in Fourier Domain for Stereoscopic X-Ray Motion Tracking
}

\author{
Dominique Zosso $^{a}$, Benoît Le Callennec ${ }^{b}$, Meritxell Bach Cuadra $^{a}$, Kamiar Aminian $^{b}$, Brigitte M. \\ Jolles $^{c}$ and Jean-Philippe Thiran ${ }^{a}$ \\ ${ }^{a}$ Ecole Polytechnique Fédérale de Lausanne (EPFL), Signal Processing Laboratory (LTS5), CH-1015 \\ Lausanne, Switzerland; \\ ${ }^{b}$ Ecole Polytechnique Fédérale de Lausanne (EPFL), Laboratory of Movement Analysis and \\ Measurement (LMAM), CH-1015 Lausanne, Switzerland; \\ ${ }^{c}$ Hôpital Orthopédique de la Suisse Romande (HOSR) and University Hospital of Lausanne (CHUV), \\ Orthopedic Department, CH-1005 Lausanne, Switzerland
}

\begin{abstract}
In this paper we present a new method to track bone movements in stereoscopic X-ray image series of the knee joint. The method is based on two different X-ray image sets: a rotational series of acquisitions of the still subject knee that allows the tomographic reconstruction of the three-dimensional volume (model), and a stereoscopic image series of orthogonal projections as the subject performs movements. Tracking the movements of bones throughout the stereoscopic image series means to determine, for each frame, the best pose of every moving element (bone) previously identified in the 3D reconstructed model. The quality of a pose is reflected in the similarity between its theoretical projections and the actual radiographs. We use direct Fourier reconstruction to approximate the three-dimensional volume of the knee joint. Then, to avoid the expensive computation of digitally rendered radiographs (DRR) for pose recovery, we develop a corollary to the 3-dimensional central-slice theorem and reformulate the tracking problem in the Fourier domain. Under the hypothesis of parallel X-ray beams, the heavy 2D-to-3D registration of projections in the signal domain is replaced by efficient slice-tovolume registration in the Fourier domain. Focusing on rotational movements, the translation-relevant phase information can be discarded and we only consider scalar Fourier amplitudes. The core of our motion tracking algorithm can be implemented as a classical frame-wise slice-to-volume registration task. Results on both synthetic and real images confirm the validity of our approach.
\end{abstract}

Keywords: Registration, X-ray, Motion analysis

\section{INTRODUCTION}

Tracking of the movements occurring at the knee joint is a prerequisite to automatized or computer-assisted movement measurements and analysis. About one million artificial knee joints are implanted every year, and almost $15^{\prime} 000$ only in Switzerland. Motion analysis is an outcome method to assess the quality of life of the patients and the functional results after a knee joint implantation, and equally allows to monitor its evolution under stress and wear. It is also performed on healthy subjects to study their specific gait and to establish general models of the movements as well. ${ }^{1-3}$

Most of the state-of-the-art methods for knee joint tracking rely on internal or external markers fixed to the different bones - mostly femur and tibia - to acquire their relative position during subject motion. ${ }^{4}$ Application of such implanted markers is however ethically arguable, in particular in healthy subjects. The goal of radiography-based pose recovery is to find the one projection of a model, that best corresponds to the measured acquisition, thus requiring computation of digitally rendered radiographs (DRR) at arbitrary angles. ${ }^{5}$ Roentgen stereophotogrammetric analysis (RSA) explores the high radiographic contrast of implanted structures to recover their position thanks to a digital model, and allows to assess the micromotion of implants with respect to the surrounding bone. ${ }^{6}$

The present study aims at the automatic recovery of the rotational movements of bones at the knee joint of subjects without implants. Our method is based on two different image sets: first, a rotational series of X-ray radiographs of the

Corresponding author: dominique.zosso@epfl.ch, Telephone: +41 21693 46 21, Fax: +41 216937600 

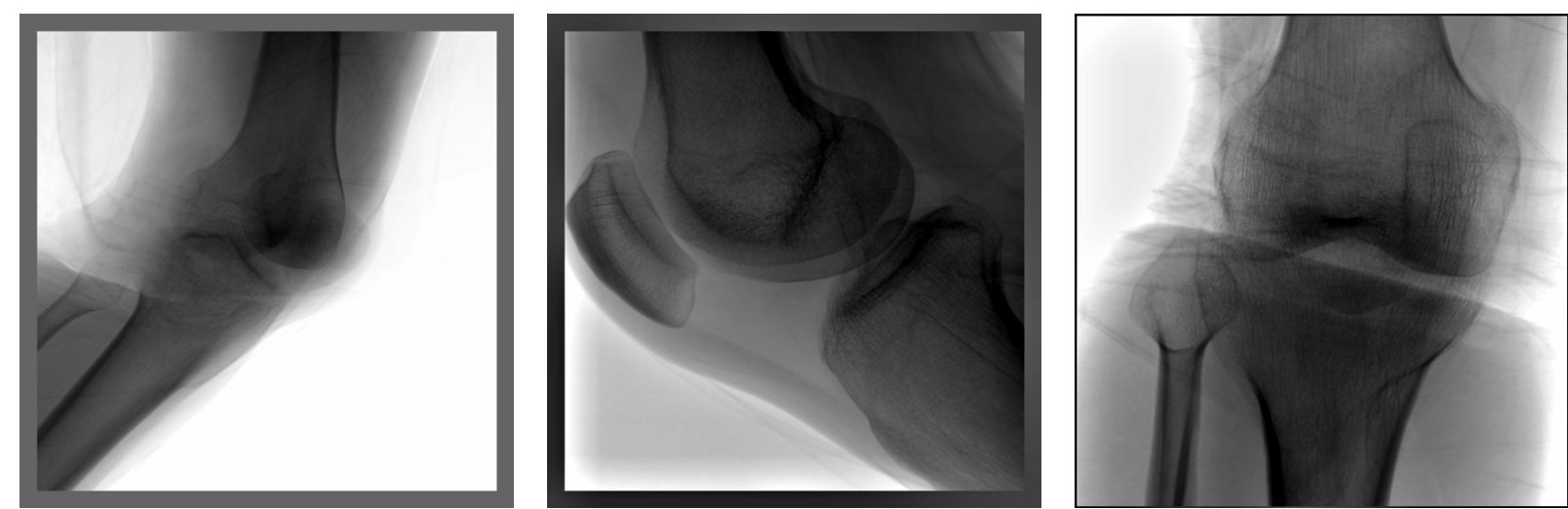

Figure 1. Left: Phase-I X-ray fluoroscopic image. One frame out of 121 in the phase-I rotational image series, illustrating the high resolution at wide field of view (FOV). Middle \& right: Lateral and frontal phase-II images. Frame 1 out of 382 of the phase-II images of the moving knee.

still subject knee, used to reconstruct its volume, and second, an orthogonal stereoscopic X-ray image series of the moving knee. The latter yields the projections used to track the bone rotations. To avoid the heavy computation of DRRs, we derive a corollary to the central-slice theorem in three dimensions. Exploring this corollary, linking the 2D Fourier transform of linear projections with central slices of the 3D Fourier volume, the projection-to-volume registration is transformed into a slice-to-volume registration in Fourier domain. This requires the projection beams be parallel. However, given the small object depth compared to the focal distance between X-ray source and receptor, we assume the - actually conic - projection beams to be "sufficiently parallel".

Section 2 on materials and methods describes the imaging setup used to acquire the two X-ray fluoroscopic image series. Further, it presents the direct Fourier method to reconstruct the 3D volume from the rotational image series, including a description of the central-slice theorem in 2D. Regarding motion tracking, we present our rigid transformation model in Fourier domain and then develop the 3D-equivalent to the 2D central-slice theorem, as well as the corollary that links the $2 \mathrm{D}$ projections with the $3 \mathrm{D}$ volume in Fourier domain. The section also describes the Gaussian masking used to isolate bones in the images and finally exposes how all these elements integrate into the registration pipeline. In section 3 we present and comment the achieved 3D reconstruction, and the motion tracking results for both an artificial tibia image series as well as the actual bi-planar fluoroscopic images. Section 4 concludes our work and ends with an outlook to future work.

\section{MATERIALS \& METHODS}

\subsection{Images}

X-ray fluoroscopic images have been acquired in the Radiology Department of the University Hospital of Lausanne (CHUV), Switzerland. The subject was lying on a bench, the knee of interest overhanging slightly to move freely. The second knee was crossed-over and torn to the body, so that it would not interfere with the imaging region of interest.

For phase-I acquisitions, rotational image series, an X-ray emitter-receiver pair, mounted on a mobile and rotating $\mathrm{C}$-arm device was used. Rotating over $210^{\circ}$ around the cranio-caudal axis, 121 images of the still, bent knee have been taken in a few seconds. Images have high resolution of $512 \times 512$ pixel and present a wide field of view (FOV), such that the distal parts of femur and proximal parts of tibia and fibula are well pictured. See figure 1 for an example.

Phase-II acquisitions need introduction of a second imaging device. Therefore, the first C-arm is brought into vertical position over the knee, acquiring frontal (dorso-ventral) images, whereas a second, horizontal C-arm yields lateral (sagittal) projections. 382 images are taken over several seconds, while the subject straightens and bends his knee twice. Phase-II images are at the same resolution as for phase-I, however, due to device limitations their FOV was reduced by a factor 2. Important collimation borders further shrink the effective image size, such that only the very parts of the bones close to the knee joint are visible. Example images are shown in figure 1. 
The radial extent of the structures of interest is more than ten times smaller than the focal distance of the C-arm device. As a consequence, the distortion introduced by the conic geometry of the X-ray beams is estimated negligible and we consider the images as being parallel projections.

\subsection{D Reconstruction}

According to Beer's Law, the observed intensity $I$ of a monochromatic X-ray beam of wavelength $\lambda$, having passed through a homogeneous object is given by:

$$
I=I_{0} \cdot e^{-\alpha_{\lambda} \rho l},
$$

where $I_{0}$ is the emission intensity, $\alpha_{\lambda}$ the attenuation factor of the material at this wavelength, $\rho$ the material density (concentration) and $l$ the path length. For inhomogeneous materials, where $\alpha_{\lambda}$ and $\rho$ become functions of the position, this law generalizes to

$$
\begin{aligned}
I & =I_{0} \cdot e^{-\int \alpha_{\lambda}(l) \rho(l) d l}, \\
-\ln \left(\frac{I}{I_{0}}\right) & =\int \alpha_{\lambda}(l) \rho(l) d l .
\end{aligned}
$$

As a consequence, the observed images $p$ of $-\ln \left(\frac{I}{I_{0}}\right)$ are the line integrals through $f(x, y, z)=\alpha_{\lambda}(x, y, z) \rho(x, y, z)$, the X-ray absorption properties of the scanned object.

\subsubsection{Radon Transform}

Under the approximation of parallel X-ray acquisition, the rotational image series $(p)$ thus represents a discrete sampling of the slice-wise cylindrical Radon transform of the scanned volume $f$, as illustrated in figure 2 (left):

$$
p_{z}(r, \theta)=\iint f_{z}\left(x^{\prime}, y^{\prime}\right) \delta\left(x^{\prime} \cos (\theta)+y^{\prime} \sin (\theta)-r\right) d x^{\prime} d y^{\prime}
$$

where each point $(r, \theta)$ is assigned the line integral of $f$ of the line normal to the radius and passing through the point. The continuous Radon transform has an exact inverse:

$$
\tilde{f}_{z}(x, y)=\iint \hat{p}_{z}(\omega, \theta)|\omega| e^{j 2 \pi \omega(x \cos (\theta)+y \sin (\theta))} d \omega d \theta
$$

where $\hat{p}_{z}(\omega, \theta)$ is the $1 \mathrm{D}$ Fourier transform in $r$ of the projection $p_{z}(r, \theta)$. The projections are ramp-filtered and backprojected over the whole image domain along the respective lines. Filtered back-projection is one of the most common tomography reconstruction techniques, although not the most efficient. ${ }^{7}$ In its general form it performs in $O\left(N^{3}\right)$.

\subsubsection{Central-Slice Theorem}

The central-slice theorem (CST) states that the Fourier transform in $r$ of a Radon projection at a given angle is equal to the central slice at the same angle of the Fourier transform of the original object:

$$
\hat{p}_{z}(\omega, \theta)=\hat{f}_{z}(\omega \cos (\theta), \omega \sin (\theta)),
$$

where $\hat{p}_{z}(\omega, \theta)$ is the 1D Fourier transform in $r$ of the projection acquired at angle $\theta$ and axial position $z$, and where $\hat{f}_{z}(u, v)$ is the in-plane 2D Fourier transform of the volume at axial position $z$. This relation is illustrated in figure 2 (right).

Proof Without loss of generality one can develop for the central slice at $\theta=0(u=\omega, v=0)$ :

$$
\begin{aligned}
\hat{f}_{z}(u, v) & =\iint f_{z}\left(x^{\prime}, y^{\prime}\right) e^{-j 2 \pi\left(u x^{\prime}+v y^{\prime}\right)} d x^{\prime} d y^{\prime} \\
& =\iint f_{z}\left(x^{\prime}, y^{\prime}\right) e^{-j 2 \pi\left(\omega x^{\prime}\right)} d x^{\prime} d y^{\prime} \\
& =\iint f_{z}\left(x^{\prime}, y^{\prime}\right) d y^{\prime} e^{-j 2 \pi\left(\omega x^{\prime}\right)} d x^{\prime} \\
& =\hat{p}_{z}(\omega, 0) .
\end{aligned}
$$




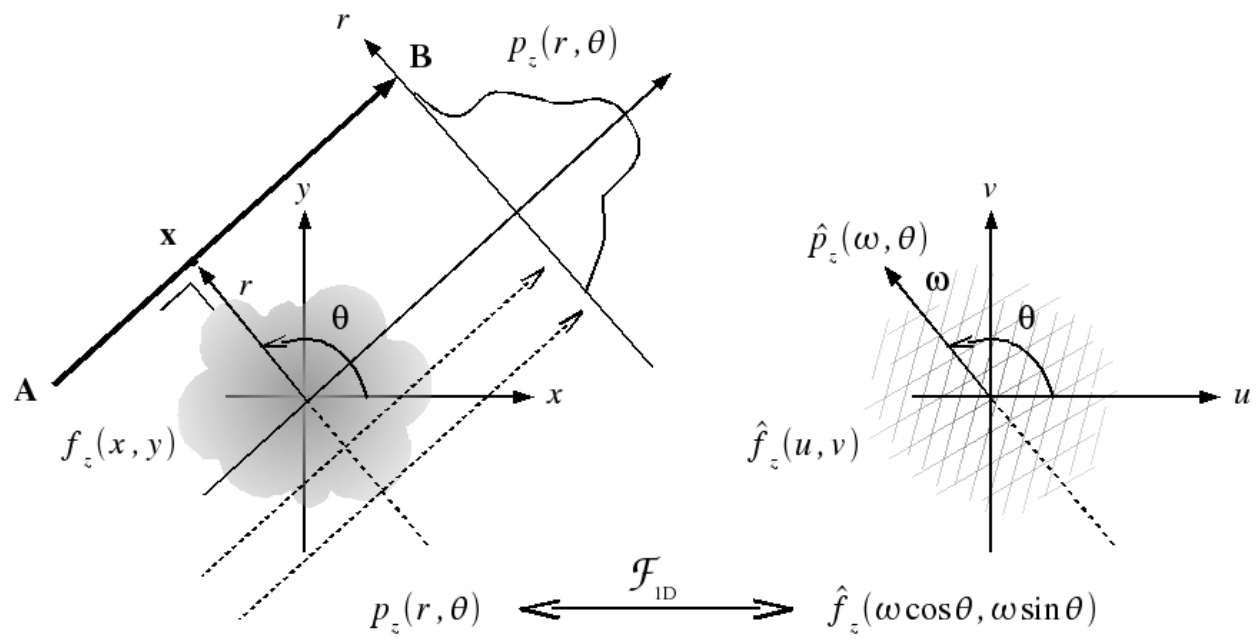

Figure 2. Left: Parallel-beam X-ray tomography. For each slice $z$, the projections $p_{z}(r, \theta)$ are made up by the line integrals from source to detector $(\mathrm{A} \rightarrow \mathrm{B})$ through the object. Right: Central-slice theorem. The 1D Fourier transform $\hat{p}_{z}(\omega, \theta)$ of a parallel projection $p_{z}(r, \theta)$ of the object $f_{z}(x, y)$, taken at an angle $\theta$ gives a slice of the 2D Fourier transform, $\hat{f}_{z}(u, v)$, subtending the same angle $\theta$ with the $u$-axis.

\subsubsection{Direct Fourier Reconstruction}

The central slice theorem allows to construct the slice-wise 2D Fourier transform in cylindrical coordinates of an object from the set of its radial projections. From the cylindrical samples the Cartesian Fourier transform can then be resampled and the volume is reconstructed by stacking the inverse Fourier transform of the $2 \mathrm{D}$ slices. This method is called direct Fourier reconstruction. ${ }^{8,9}$ The basic form of the algorithm performs in $O\left(N^{2} \log (N)\right)$, as imposed by the inverse 2D FFT. Its most critical step is the polar to Cartesian resampling: While the polar sample density is high in the lower frequency band, information gets sparse towards the higher frequencies. ${ }^{10}$ Zero-padding of the projections and oversampling of the Cartesian 2D Fourier transform with an appropriate interpolation scheme allows to reduce artifacts. ${ }^{11}$

A publicly available implementation ${ }^{12}$ of the direct Fourier reconstruction method has been used to reconstruct a 3D model from the rotational image series within the Insight Segmentation and Registration Toolkit (ITK). ${ }^{13}$ The implementation and parameters of the direct Fourier reconstruction method have extensively been tested and discussed at publication. In the present context we chose a zero-padding factor $n_{z}=2,2 \mathrm{D}$-DFT oversampling rate $n_{g}=2$ and cubic b-spline interpolation in Fourier domain.

\subsection{Motion Tracking}

In this context we achieve bone motion tracking by frame-wise pose estimation of the 3D model based on the bi-planar $\mathrm{X}$-ray views (tracking by detecting). For each frame, the rigid bone model is aligned with the bi-planar projections, i.e. one is looking for those spatial transform parameters that best explain the observed projections. Motion is then represented as the evolution of those transform parameters over the whole image sequence.

To avoid the lengthy computations of digitally rendered radiographs (DRR), we want to reformulate the projectionsto-volume registration in spatial domain as a slice-to-volume registration in Fourier domain. The necessary elements are established in the following paragraphs, i.e. the equivalent of a rigid spatial transformation in Fourier domain, a corollary to the central-slice theorem in 3D, that allows to identify 2D Fourier transforms of linear projections with central slices of the 3D Fourier volume, and Gaussian masking prior to Fourier transformation to isolate different bones. We present how these building blocks integrate into a complete registration pipeline and finally describe a simple test method to assess the outcome of our method. 


\subsubsection{Spatial Transformation in Fourier Domain}

The Fourier domain approach requires knowledge of the impact of a rigid spatial transformation in Fourier domain. Let $f_{1}$ be a reference image and $f_{2}$ its rigidly transformed version:

$$
f_{2}(\mathbf{x})=f_{1}(\mathbf{R}(\mathbf{x}+\Delta \mathbf{x})),
$$

with $\mathbf{R}$ the rotation matrix and $\Delta \mathbf{x}$ the translation vector. The Fourier transform of $f_{2}$ then writes: ${ }^{14}$

$$
\begin{aligned}
\hat{f}_{2}(\omega) & =\int f_{2}(\mathbf{x}) e^{-j 2 \pi \omega^{T} \mathbf{x}} d \mathbf{x}=\int f_{1}(\mathbf{R}(\mathbf{x}+\Delta \mathbf{x})) e^{-j 2 \pi \omega^{T} \mathbf{x}} d \mathbf{x} \\
& =e^{j 2 \pi \omega^{T} \Delta \mathbf{x}} \int f_{1}\left(\mathbf{R} \mathbf{x}^{\prime}\right) e^{-j 2 \pi \omega^{T} \mathbf{x}^{\prime}} d \mathbf{x}^{\prime}=e^{j 2 \pi \omega^{T} \Delta \mathbf{x}} \int f_{1}\left(\mathbf{x}^{\prime \prime}\right) e^{-j 2 \pi(\mathbf{R} \omega)^{T} \mathbf{x}^{\prime \prime}} d \mathbf{x}^{\prime \prime} \\
& =e^{j 2 \pi \omega^{T} \Delta \mathbf{x}} \hat{f}_{1}(\mathbf{R} \boldsymbol{\omega}),
\end{aligned}
$$

with $\mathbf{x}^{\prime}=\mathbf{x}+\Delta \mathbf{x}$ and $\mathbf{x}^{\prime \prime}=\mathbf{R} \mathbf{x}^{\prime}$. While the rotation in signal domain corresponds to the same rotation in Fourier domain (rotation invariance), the translation manifests itself as a phase shift. Thus:

$$
\left|\hat{f}_{2}(\omega)\right|=\left|\hat{f}_{1}(\mathbf{R} \omega)\right|
$$

For the present study, only rotations are of interest. We discard the translation-relevant phase information in Fourier domain and evaluate pose quality based on frequency amplitudes only. This reduction offers important simplifications as the Fourier transforms are computed once and no ulterior phase updates are required during registration. Further, the considered Fourier images become scalar and conventional image metrics are applicable.

In many applications, including the present context, rotations are not represented as matrices, but rather as unit quaternions. The four components of a unit quaternion are $\mathbf{x}=x_{0}+i \cdot x_{1}+j \cdot x_{2}+k \cdot x_{3}$, where $x_{1} \ldots x_{3}$ span the rotation axis. The rotation angle is defined by $\alpha=2 \cos ^{-1}\left(x_{0}\right)$. As a unit quaternion has only three degrees of freedom, $x_{1} \ldots x_{3}$ usually are selected as the free parameters, and $x_{0}$ is inferred to obtain the unit norm. The rotation angle can therefore be calculated directly from the three free parameters as $\alpha=2 \sin ^{-1}\left(\sqrt{x_{1}^{2}+x_{2}^{2}+x_{3}^{2}}\right)$. The zero-length rotation axis $\left(x_{0}=1, x_{1}=x_{2}=x_{3}=0\right)$ corresponds to the identity transform $(\alpha=0)$.

\subsubsection{Central-Slice Theorem in 3D}

In three dimensions, the Radon transform assigns to each point $\mathbf{x}=(x, y, z)$ the plane integral normal to the radial vector $\mathbf{x}$ and passing through that point:

$$
R(\mathbf{x})=\int f\left(\mathbf{x}^{\prime}\right) \boldsymbol{\delta}\left(\mathbf{x} \cdot\left(\mathbf{x}^{\prime}-\mathbf{x}\right)\right) d \mathbf{x}^{\prime}
$$

where $R(\mathbf{x})$ denotes the Radon transform of $f$ in three dimensions. In spherical coordinates, with $x=r \cos (\theta) \sin (\phi)$, $y=r \sin (\theta) \sin (\phi)$ and $z=r \cos (\phi)$, this writes:

$$
R(r, \theta, \phi)=\iiint f\left(x^{\prime}, y^{\prime}, z^{\prime}\right) \delta\left(x^{\prime} \cos (\theta) \sin (\phi)+y^{\prime} \sin (\theta) \sin (\phi)+z^{\prime} \cos (\phi)-r\right) d x^{\prime} d y^{\prime} d z^{\prime} .
$$

The equivalent to the central-slice theorem in three dimensions then states that the Fourier transform in $r$ of a Radon projection at given angles is equal to the central line at the same angles of the Fourier transform of the original object:

$$
\hat{R}(\omega, \theta, \phi)=\hat{f}(\omega \cos (\theta) \sin (\phi), \omega \sin (\theta) \sin (\phi), \omega \cos (\phi)),
$$

where $\hat{R}(\omega, \theta, \phi)$ is the 1D Fourier transform in $r$ of the Radon transform at angles $\theta$ and $\phi$, and where $\hat{f}(u, v, w)$ is the 3D Fourier transform of the volume. 
Proof Without loss of generality one can develop for the central line at $\theta=0, \phi=\pi / 2(u=\omega, v=w=0)$ :

$$
\begin{aligned}
\hat{f}(u, v, w) & =\iiint f\left(x^{\prime}, y^{\prime}, z^{\prime}\right) e^{-j 2 \pi\left(u x^{\prime}+v y^{\prime}+w z^{\prime}\right)} d x^{\prime} d y^{\prime} d z^{\prime} \\
& =\iiint f\left(x^{\prime}, y^{\prime}, z^{\prime}\right) e^{-j 2 \pi\left(\omega x^{\prime}\right)} d x^{\prime} d y^{\prime} d z^{\prime} \\
& =\iiint f\left(x^{\prime}, y^{\prime}, z^{\prime}\right) d y^{\prime} d z^{\prime} e^{-j 2 \pi\left(\omega x^{\prime}\right)} d x^{\prime} \\
& =\hat{R}(\omega, 0, \pi / 2) .
\end{aligned}
$$

In the actual problem we are not dealing with plane integrals in $3 \mathrm{D}$, and therefore need to further develop the theorem.

\section{Corollary: The 2D Fourier transform of a linear projection through a volume identifies with the central slice through the 3D Fourier transform of that volume, at the same angles.}

Proof Let $\Sigma$ be the (central) imaging plane corresponding to an acquired 2D image $p_{\theta}$, projection of the original object $f$, where for simplicity and taking the cylindrical acquisition geometry into account, $\Sigma$ contains the $z$-axis and be defined by $\theta$ only, as illustrated in figure 3 . This image $p_{\theta}$ is the set of the line integrals through $f$ normal to $\Sigma$. Therefore a plane integral of $f$ in any plane $\Lambda$ normal to $\Sigma$ can be obtained by a line integral of $p_{\theta}$ along the intersection $\Lambda \cap \Sigma$. As such, the 3-dimensional Radon transform of $f, R(\mathbf{x}), \mathbf{x}(r, \theta, \phi) \in \Sigma$, can be expressed as a 2-dimensional Radon transform of the projection $p_{\theta}$ as follows:

$$
R(r, \theta, \phi)=R\left[p_{\theta}\left(x^{\prime}, y^{\prime}\right)\right](r \sin (\phi), r \cos (\phi))=\iint p_{\theta}\left(x^{\prime}, y^{\prime}\right) \delta\left(x^{\prime} \sin (\phi)+y^{\prime} \cos (\phi)-r\right) d x^{\prime} d y^{\prime},
$$

where $p_{\theta}\left(x^{\prime}, y^{\prime}\right)$ is the projection acquired at angle $\theta$. Let $\hat{p}_{\theta}(u, v)$ be the 2D Fourier transform of the projection $p_{\theta}$. Therefore, using the 2D CST one can write:

$$
\hat{R}(\omega, \theta, \phi)=\hat{p}_{\theta}(\omega \sin (\phi), \omega \cos (\phi))=\hat{f}(\omega \cos (\theta) \sin (\phi), \omega \sin (\theta) \sin (\phi), \omega \cos (\phi)),
$$

where (12) has been used to write the last equality.

Alternatively, the corollary can also be shown straightforwardly (for simplicity be $\theta=0: u=\omega \sin (\phi), v=0, w=$ $\omega \cos (\phi))$ :

$$
\begin{aligned}
\hat{f}(u, v, w) & =\iiint f\left(x^{\prime}, y^{\prime}, z^{\prime}\right) e^{-j 2 \pi\left(u x^{\prime}+v y^{\prime}+w z^{\prime}\right)} d x^{\prime} d y^{\prime} d z^{\prime} \\
& =\iiint f\left(x^{\prime}, y^{\prime}, z^{\prime}\right) e^{-j 2 \pi\left(u x^{\prime}+w z^{\prime}\right)} d x^{\prime} d y^{\prime} d z^{\prime} \\
& =\iiint f\left(x^{\prime}, y^{\prime}, z^{\prime}\right) d y^{\prime} e^{-j 2 \pi\left(u x^{\prime}+w z^{\prime}\right)} d x^{\prime} d z^{\prime} \\
& =\hat{p}_{\theta=0}(\omega \sin (\phi), \omega \cos (\phi)) .
\end{aligned}
$$

\subsubsection{Masking}

Motion tracking requires determining the pose that best explains the observed orthogonal projections, for all moving elements of the scene and in all frames of the stereoscopic X-ray image series. Appropriate masking allows to determine the optimal pose of each element individually. However, this step is particularly challenging as several critical complications interfere, in particular several structures could overlap in the projections of the knee joint. The stereoscopic X-ray acquisitions at hand have a very limited field of view due to the used collimators. Both image boundaries and masking create artifacts in Fourier domain that might degrade traceability. We chose uniform Gaussian masking as a compromise offering good selectivity between different image elements and boundaries, while still limiting Fourier domain artifacts. 


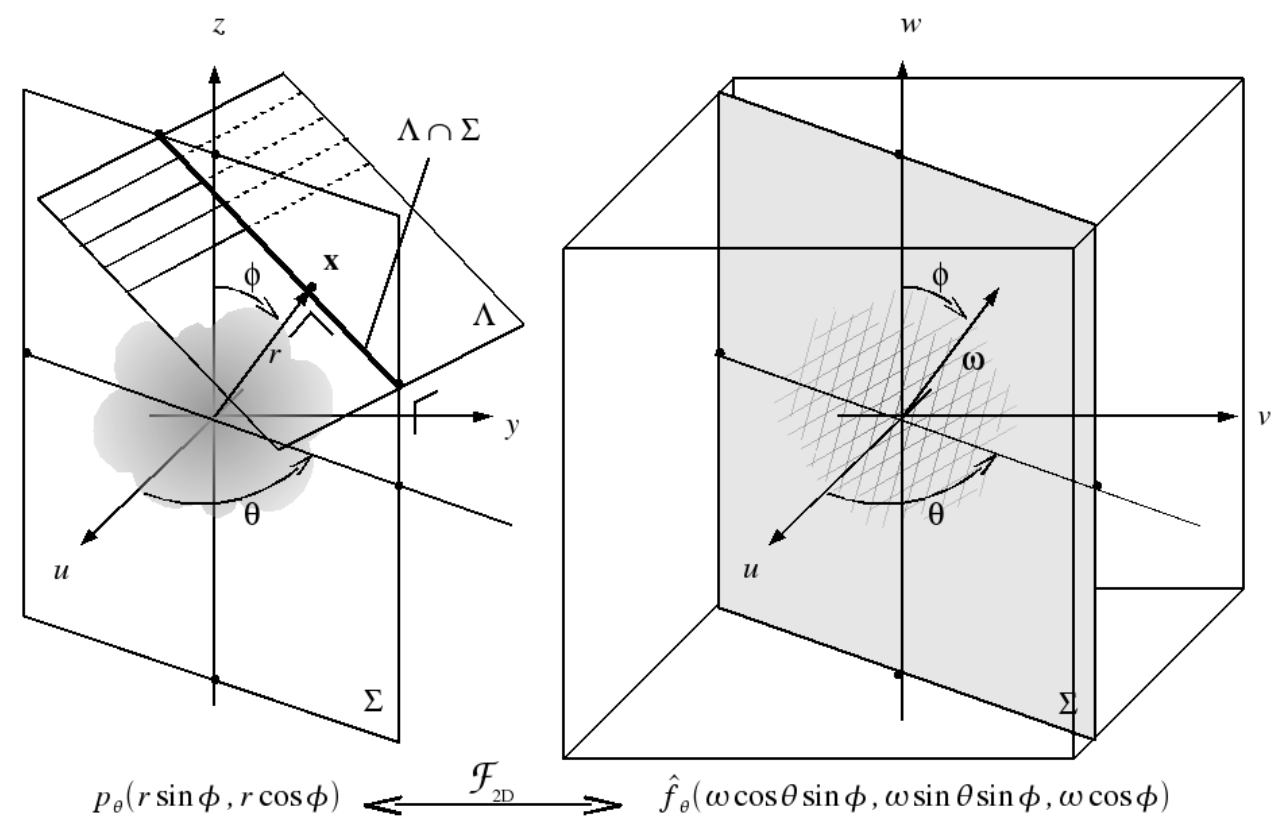

Figure 3. Left: $3 D$ Radon transform. For each point $\mathbf{x}$, the 3D Radon transform is defined by the plane integral of $f$ in $\Lambda$. The plane integral can be split into a line integral along $\Lambda \cap \Sigma$ of the linear projections $p_{\Sigma}$, which in turn are line integrals in $\Lambda$, and where $\Sigma \perp \Lambda$ is the central imaging plane of projections. Right: Corollary to the $3 D$ central-slice theorem. The 2D Fourier transform $\hat{p}_{\theta}(\omega \sin (\phi), \omega \cos (\phi))$ of a parallel projection $p_{\theta}$ of an image $f(x, y, z)$ taken at an angle $\theta$ gives a slice in the 3D Fourier transform volume, $\hat{f}(u, v, w)$, subtending the same angle $\theta$ with the $u$-axis.

By the convolution theorem, multiplication with a Gaussian in signal space equals convolution with its transform in Fourier domain. Masking in signal space can simply be considered as smoothing in Fourier domain, preceded and followed by a phase shift to account for the center of the Gaussian mask:

$$
y(\mathbf{x})=f(\mathbf{x}) g\left(\mathbf{x}-\mathbf{x}_{0}\right) \Longleftrightarrow \hat{y}(\omega)=\hat{f}(\omega) *\left(e^{-j \omega^{T} \mathbf{x}_{0}} \hat{g}(\omega)\right)=e^{-j \omega^{T} \mathbf{x}_{0}}\left(\left(e^{j \omega^{T} \mathbf{x}_{0}} \hat{f}(\omega)\right) * \hat{g}(\omega)\right),
$$

where $f$ is the original image, $g$ a Gaussian mask, $\mathbf{x}_{0}$ its center, and $*$ denotes convolution. The spread of $\hat{g}$ is inverse to the spread of $g$ - the smaller the mask the more important the smoothing in Fourier domain. Center and spread of the Gaussian mask have to be defined carefully to ensure good coverage of the bone structures of interest throughout the whole image series.

\subsubsection{D-to-3D Registration}

A schematic representation of the registration setup is given in figure 4. Rotation invariance of the Fourier transform allows to look for the rotation that links two images in Fourier domain. While computation of the 3D discrete Fourier transform (3D DFT) of the model is straightforward, the corollary to the 3D central-slice theorem yields two orthogonal central slices through the virtual 3D DFT from the bi-planar projections of phase II. Pose recovery can therefore be formulated as registration of the orthogonal pair of 2D DFT of phase-II projections with the DFT volume built after phase I, with centered rotation as rigid transform model. Centered rotation is represented as unit quaternions with 3 degrees of freedom, i.e. $x_{1} \ldots x_{3}$, the rotation axis vector components, the norm of which determines the rotation angle. Eventually, motion tracking is the evolution of these transform parameters through all frames.

For each bone that is to track individually, the phase-I 3D model and all phase-II projections are masked using a multiplicative Gaussian mask. The position of the mask in the phase-II images is fixed by hand to an appropriate position and size to allow for visibility of the necessary structures in all frames. The masked model is then statically transformed into Fourier domain using a fast Fourier transform (FFT). For each frame, the masked stereoscopic projections are transformed into Fourier domain using 2D FFT. Only the amplitude is considered as to discard any information on translation. Resulting images are smoothed using a Gaussian kernel to flatten the metric space and enlarge the capture-range. 


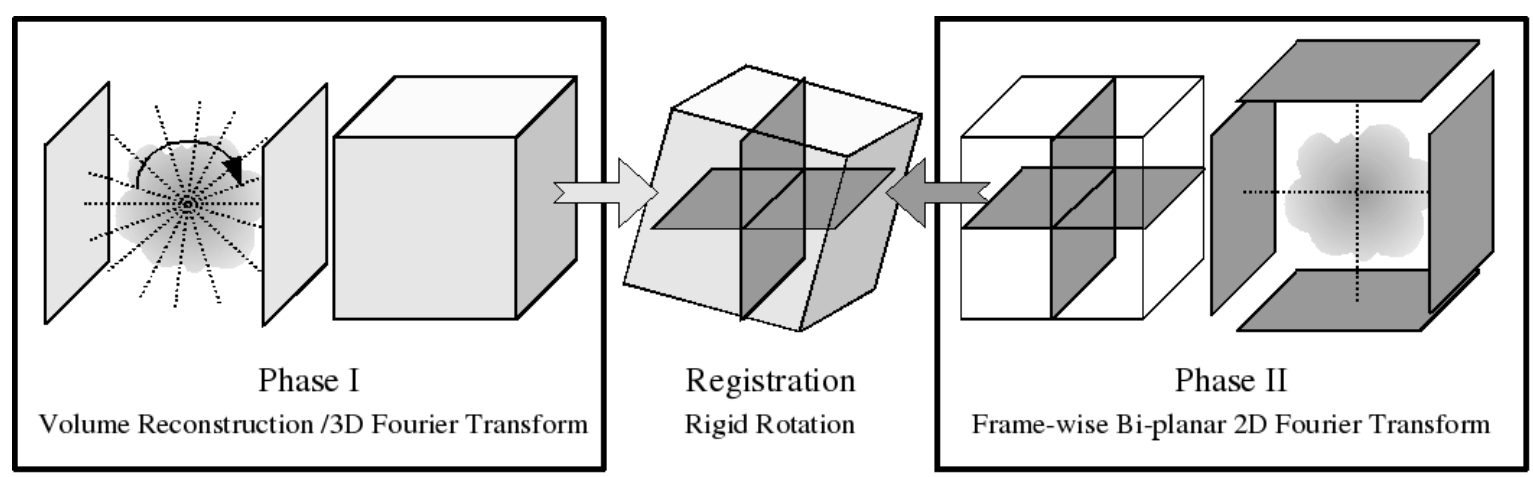

Figure 4. Bi-planar 2D-to-3D registration scheme. Phase-I rotational images are reconstructed into a 3D model and its 3D Fourier transform. Phase-II bi-planar projections are transformed into a pair of Fourier slices using the corollary to the 3D central-slice theorem. Fourier volume and slices are registered for each frame, to retrieve the rotation parameters that link phase-I with phase-II objects. Evolution of those parameters equals motion tracking.
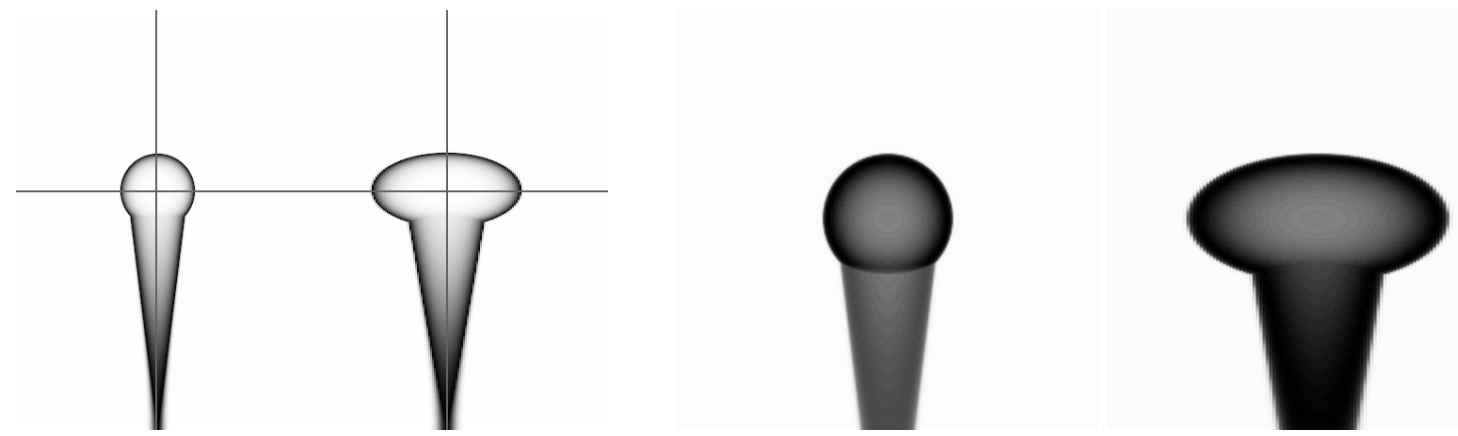

Figure 5. Left: Lateral and frontal section through virtual tibula. Right: Lateral and frontal projections of virtual tibula. Images have similar field of view as the corresponding actual fluoroscopy acquisitions. They are noise-free and do not contain further bone structures.

Sequentially, each frame is registered with the static model. A gradient descent optimizer tunes the parameters of a centered rotation transform to recover the best alignment of the orthogonal 2D Fourier image pair within the static 3D Fourier volume. The quality of a pose, i.e. the similarity between slices and their volumetric counterpart, is evaluated using Mattes' mutual information metric. ${ }^{15}$ To take advantage of the high correlation between subsequent frames, registration results (i.e. transform parameters) are propagated as initialization to the subsequent frame registration.

\subsubsection{Phantom Image}

Prior to registering the fluoroscopic images, the registration pipeline is tested on artificial images. A simple phantom image representing a virtual tibia has been built, as shown in figure 5. Projections have been calculated without any bone movement.

The test setup consists of launching a series of registration tasks with transform initializations differing from identity. Random samples of the transform space are selected as initialization to the registration task. Registration is considered successful if the parameters converge to the identity transform, i.e. the norm of the rotation axis vector converges to 0 . 


\section{RESULTS \& DISCUSSION}

\subsection{D Reconstruction}

Direct Fourier reconstruction method has successfully been applied to the rotational image series. The results of the 3D reconstruction of phase-I images are illustrated in figure 6.

The reconstructed phase-I model exhibits high quality close to the $z$-axis. Some degradation in form of blurring becomes apparent further away. This is a direct consequence of the conic X-ray scan geometry and demonstrates the limits of the parallel-beam approximation. As the important structures are located around the $z$-axis, outward image degradation does not represent a serious issue.

The white disk is the image of the collimator boundaries in the phase-I acquisitions. Zero-padding outside the collimator borders causes high-frequency artifacts around the disk borders in the reconstruction. Vanishing abrupt boundaries in the phase-I images, and padding with the correct background intensity would attenuate those artifacts. Further, all images of phase I should picture the same content. The radial extent of the FOV is not sufficient to acquire the distal parts of the lower limb in sagittal projections, whereas the same parts have been scanned in coronal views. Although the region of interest (ROI) is sufficiently well represented, reconstruction quality would be improved when taking these constraints into account for further acquisitions.

\subsection{Motion Tracking}

The whole registration pipeline has been implemented in $\mathrm{C}++$, using the Insight Segmentation and Registration Toolkit (ITK). ${ }^{13}$ Registration results obtained on phantom images and experimental data are described in the following paragraphs.

\subsubsection{Phantom Image}

More than 800 random seeds have been selected to test the performance of the bi-planar registration pipeline on the phantom image. Unit quaternion parameters $x_{1} \ldots x_{3}$ are independent realizations of an exponentially distributed random variable with mean $\lambda=0.05$. This leads to a denser sampling of the transform parameter space close to identity and within the presumed capture range.

Depending on the seed position, registration converges towards different attractors. The most important attractor is the origin of the transform space, corresponding to identity. As illustrated in figure 7 (left), it is correctly attained by all registration runs launched within $6^{\circ}$, corresponding to a norm of the seed rotation axis smaller than 0.0523 . The capturerange is almost isotropic, and is therefore represented as a spherical shell. The domain of transition from successful convergence to misregistration is narrow: beyond $11^{\circ}$ no successful capture could be observed, as shown in the capture histogram in figure 7 (right).

Making use of the phantom symmetry, the parameter space was sampled in the positive octant only. As optimization usually stops before zero-norm is exactly attained (convergence criteria), the observed attractor is biased. Nevertheless, misregistration is negligible, as the mean of the results considered successful is located at $\left(x_{1}, x_{2}, x_{3}\right)=(0.069,0.462,0.027)$. $10^{-3}$, corresponding to a rotation angle of less than $0.054^{\circ}$, with a standard deviation along the principal directions of $\sigma=(0.004,0.046,0.149) \cdot 10^{-3}$.

Capture-range essentially depends on the image content: unspecific structures in Fourier domain, noise and artifacts will severely degrade robustness. Further, a well-placed mask is critical: if projections and model are not masked to roughly the same structures, registration will be misled.

\subsubsection{Real Data}

After reconstructing the volume of the subject knee based on the phase-I images, we applied the proposed motion tracking method to automatically recover the rotations of the tibia throughout all phase-II images. Two sample data-set frames of straight and bent knee configuration, and the contours of the applied masks are illustrated in figure 8 (left).

As a simple way of verification, a centroid reference line has been manually identified in the 3D model. The same center-line has manually been located in every tenth frame of the stereoscopic image series. A comparison between the evolution of both the manually sampled and the automatically tracked center-line angle with respect to its initial position is shown in figure 8 (right). 
For the algorithm to succeed with a fixed mask, a medium sized mask configuration had to be chosen. This is a compromise between a small mask allowing to consider information close to the image boundaries while limiting the algorithmic field of view, and a big mask size pushing the mask center further away from the collimator borders and complicating convergence in bent position. The deflection plot indicates a very good tracking quality except for the first frames in bent configuration, where important structures of the tibia are situated in the immediate vicinity of the image boundaries. The remaining differences between manual and automatic tracking are also in part explained by the limited precision of the manual tracking.

The total amount of motion of the tibia from bent to straight position is close to $45^{\circ}$, as indicated by the centerline deflection amplitude. This exceeds by far the capture-range of the registration algorithm observed with the phantom image. However, propagation of the registration results as initialization to the next frame allows to considerably reduce the registration effort. Meanwhile, wrong registration results get propagated to the next frame as well, and the algorithm gets stuck when caught in off-track optima. This happens in the first few frames, where the algorithm first fails to get on track. On the other hand, if registration is performed in reverse direction out of a successfully registered frame, tracking can succeed, as illustrated by the dotted line in 8 (right).

\section{CONCLUSIONS \& OUTLOOK}

We proposed a method for motion tracking in stereoscopic X-ray imaging of the knee joint that is, to the best of our knowledge, novel in the field. The approach uses rotational fluoroscopic images of the still knee to reconstruct a 3dimensional model by direct Fourier reconstruction. A second image series of orthogonal projections through the moving knee forms the basis for motion tracking.

We presented a corollary to the central-slice theorem in three dimensions, that states that the 2D Fourier transform of linear projections through an object is equal to a slice in the corresponding 3D Fourier volume. The corollary allowed to avoid the lengthy computations of digitally rendered radiographs and to reformulate the projection-volume registration as a less complex Fourier domain slice-volume registration. Reducing to angular tracking and considering Fourier amplitudes only, allowed to apply classical image registration techniques with a global rotation transform model: registration looked for the transform parameters producing the best alignment of the orthogonal Fourier slices with the Fourier volume.

The registration pipeline further used appropriate masking by Gaussian masks in spatial domain to isolate the different bones and track them individually. Transform parameters determined for one frame were propagated as initialization to the next frame. Motion tracking finally is the evolution of these rotation parameters through the whole bi-planar image series.

Our method has been tested with success on an artificial tibia phantom image. Application to the real world data yielded plausible results, which were confirmed by data extracted using manual tracking. We are currently applying the tracking algorithm to other bones related to the knee joint to complete the set of rotations and to provide detailed information about the evolution of relative angles. As the study will be extended to other healthy and pathological subjects, our algorithm will run on further data-sets and provide complementary information about its robustness on real data.

The pilot study results obtained in the present work will also allow to improve the setup for upcoming acquisitions. Several shortcomings of the current images have been pointed out, e.g. the incoherent FOV of phase-I images (i.e. some structures not pictured under all angles), or the tight FOV of the bi-planar projections in phase II.

The slice-to-volume registration method in Fourier domain might be an efficient solution of rotational motion tracking in quasi-parallel projections in other application fields. This covers the wide spectrum of interventional X-ray imaging of rigid structures. Extension to non-parallel geometry and inclusion of translation is feasible. 


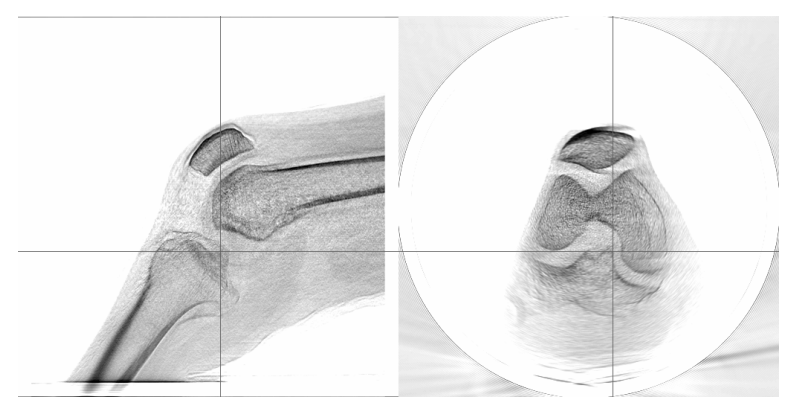

Figure 6. Sagittal (left) and transverse (right) slice through the raw reconstructed $3 D$ volume of the knee joint. The vertical cursors indicate the respective position of the slices. The cross-section illustrates how important the bones (patella, femur and tibia) are interdigitated at the knee joint, complicating their isolated masking.
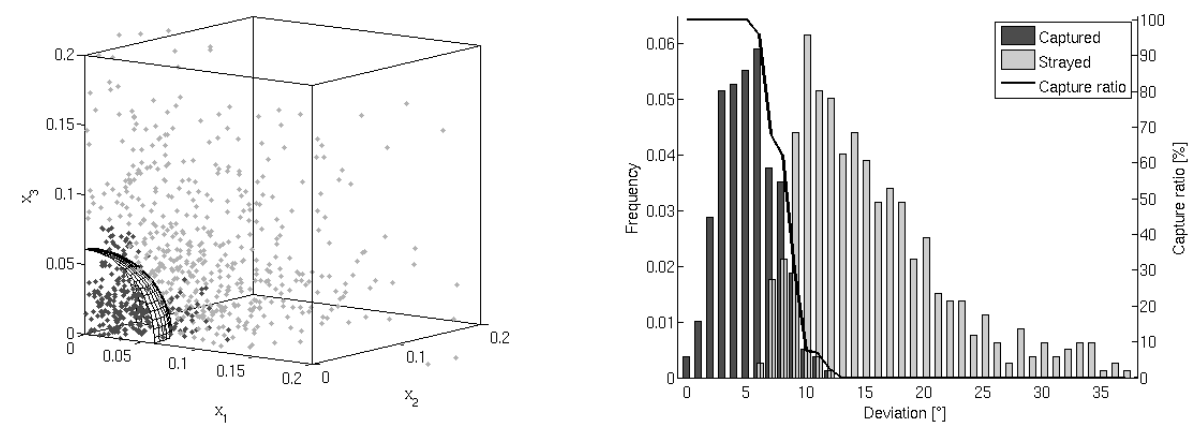

Figure 7. Left: Capture-range of rotation transform seeds. The three axes correspond to the three unit quaternion components $x_{1}$, $x_{2}$ and $x_{3}$. Dark spots represent successfully registered transform seeds, light spots have failed. The spherical mesh, enveloping only successful seeds, illustrates the estimated capture range of $6^{\circ}$. Right: Capture histogram over rotation angle initialization. Dark bars show the frequency of successful attempts depending on initial deviation angle, light bars correspond to failure. The solid line indicates the success-to-failure ratio in every histogram bin.
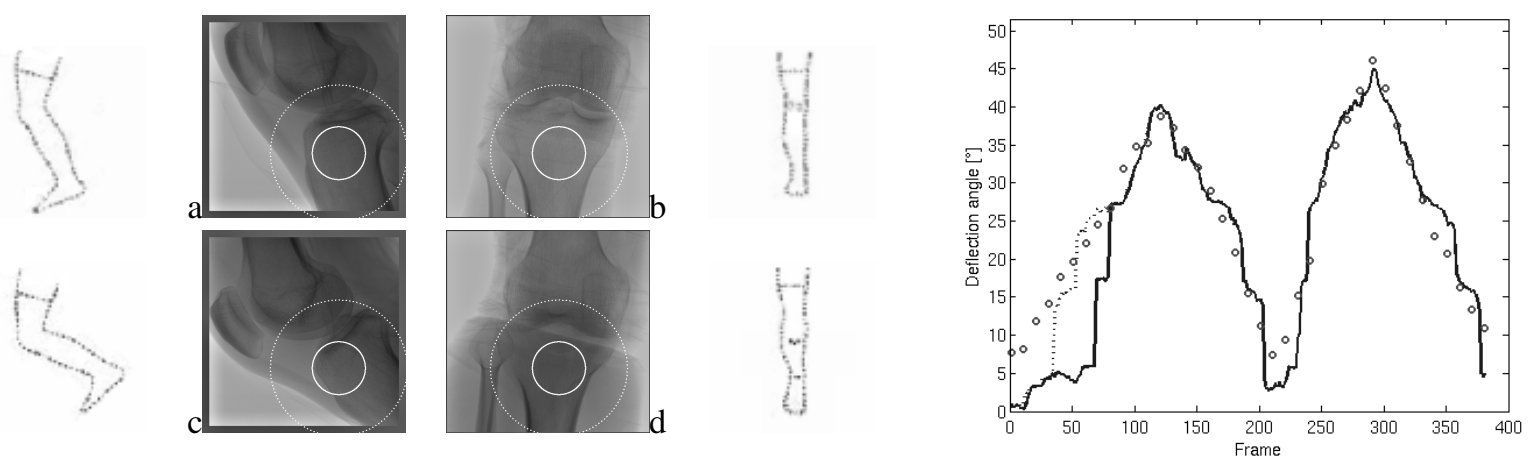

Figure 8. Left: Lateral $(a+c)$ and frontal $(b+d)$ images of the stereoscopic X-ray acquisitions of the knee at straight $(a+b)$ and bent position $(c+d)$. The solid circles correspond to the half-maximum width of the Gaussian mask for tibia-tracking; dashed circles represent its $1 \%$ contour line. The lateral images reveal the extremely tight collimator FOV and the frontal images show the overlapping of bones in the central region. Right: Comparison between automatic and manual tracking of tibia. The plot shows the evolution of the deflection angle of a reference line as automatically detected (solid line) and as extracted by hand for every 10th frame (circles). The subject extends and bends the knee twice. Tracking quality is problematic in bent position, but reverse processing allows to improve tracking quality for frames $32 \ldots 80$ (dotted line). 


\section{ACKNOWLEDGMENTS}

The authors are grateful to Julien Chardonnens from LMAM, Nicolas Theumann and Hervé Martin of the CHUV radiology department for their precious collaboration for $\mathrm{X}$-ray acquisitions.

This work has been supported by the Center for Biomedical Imaging (CIBM) of the Geneva - Lausanne Universities, the EPFL, as well as the foundations Leenaards and Louis-Jeantet.

\section{REFERENCES}

1. K. Aminian, C. Trevisan, B. Najafi, H. Dejnabadi, C. Frigo, E. Pavanc, A. Telonio, F. Cerati, E. Marinoni, P. Robert, and P.-F. Leyvraz, "Evaluation of an ambulatory system for gait analysis in hip osteoarthritis and after total hip replacement," Gait and Posture 20, pp. 102-107, 2004.

2. H. Dejnabadi, B. Jolles, E. Casanova, P. Fua, and K. Aminian, "Estimation and visualization of sagittal kinematics of lower limbs orientation using body fixed sensors," IEEE Transactions on Biomedical Engineering 53(7), pp. 13821393, 2006.

3. H. Dejnabadi, B. Jolles, and K. Aminian, "A new approach for quantitative analysis of inter-joint coordination during gait," IEEE Transactions on Biomedical Engineering . in press.

4. E. H. Garling, B. L. Kaptein, K. Geleijns, R. G. Nelissen, and E. R. Valstar, "Marker Configuration Model-Based Roentgen Fluoroscopic Analysis," Journal of Biomechanics 38, pp. 893-901, 2005.

5. L. Zöllei, E. Grimson, A. Norbash, and W. Wells, "2D-3D rigid registration of X-ray fluoroscopy and CT images using mutual information and sparsely sampled histogram estimators," in Proceedings of the 2001 IEEE Computer Society Conference on Computer Vision and Pattern Recognition, CVPR2001, 2, pp. 696-703, 2001.

6. E. Valstar, F. de Jong, H. Vrooman, P. Rozing, and J. Reiber, "Model-based Roentgen stereophotogrammetry of orthopaedic implants," Journal of Biomechanics 34, pp. 715-722, jun 2001.

7. R. M. Lewitt, "Reconstruction Algorithms: Transform Methods," in Proceedings of the IEEE, 71, pp. 390-408, march 1983.

8. H. Stark, J. Woods, I. Paul, and R. Hingorani, "Direct Fourier reconstruction in computer tomography," IEEE Transactions on Acoustics, Speech, and Signal Processing [see also IEEE Transactions on Signal Processing] 29, pp. 237245, April 1981.

9. D. Gottlieb, B. Gustafsson, and P. Forssén, "On the Direct Fourier Method for Computer Tomography," IEEE Transactions on Medical Imaging 19, pp. 223-232, march 2000.

10. H. Stark, "Sampling theorems in polar coordinates," Journal of the Optical Society of America 69, pp. 1519-1525, nov 1979.

11. M. Magnusson, P.-E. Danielsson, and P. Edholm, "Artefacts and Remedies in Direct Fourier Tomographic Reconstruction," Nuclear Science Symposium and Medical Imaging Conference 2, pp. 1138-1140, october 1992.

12. D. Zosso, M. Bach Cuadra, and J.-P. Thiran, "Direct fourier tomographic reconstruction image-to-image filter," Insight Journal , July-December 2007.

13. L. Ibanez, W. Schroeder, L. Ng, and J. Cates, The ITK Software Guide. Kitware, Inc., http://www.itk.org/ItkSoftwareGuide.pdf, second ed., 2005.

14. P. Vandewalle, S. Süsstrunk, and M. Vetterli, "A Frequency Domain Approach to Registration of Aliased Images with Application to Super-Resolution," EURASIP Journal on Applied Signal Processing (special issue on Superresolution) 2006, pp. 1-14, 2006.

15. D. Mattes, D. Haynor, H. Vesselle, T. Lewellen, and W. Eubank, "Non-rigid Multimodality Image Registration," in Proceedings SPIE, Medical Imaging 2001: Image Processing, 4322, pp. 1609-1620, 2001. 